\title{
MEASUREMENT OF THREE-DIMENSIONAL FLUCTUATING LIQUID VELOCITIES*
}

\author{
SHIRo ITO, SHOJI URUSHIYAMA** AND KoHeI OGAWA \\ Department of Chemical Engireering, Tokyo Institute of \\ Technology, Tokyo
}

\begin{abstract}
For measuring three-dimensional turbulence velocities, a new technique based on electrochemical reaction controlled by mass transfer rate is developed. The measuring probe for this method consists of a plastic sphere in which more than three polarized electrodes are plugged, their surfaces flush with the sphere surface. By making use of this measuring probe threedimensional turbulence properties can be measured more correctly. An example of the application of this method for measurement of flow in a stirred vessel is shown.
\end{abstract}

\section{Introduction}

For studying the phenomena occurring in chemical process equipment of flow systems, it is necessary to know not only the three-dimensional mean velocities but also the three-dimensional turbulence properties, that is, turbulent intensities, double and triple velocity correlations, etc. Hot-wire anemometers have been widely used as a measuring instrument for gas flow systems. However, from the standpoint of stability this method may be less suitable for measuring liquid velocity.

For measuring the velocity of three-dimensional steady liquid flow, a new technique based on electrochemical reaction controlled by mass transfer rate was developed in our laboratory as previously reported $^{2}$. The measuring probe for the above method consists of a plastic sphere in which a small polarized circular electrode is plugged. Its surface is flush with the sphere surface, as shown in Fig. 1. In the above measuring method the diffusional current between the small electrode on the sphere and the opposite electrode located in the electrolyte depends on the liquid velocity and the angle between the direction of liquid flow and the radius through the center of a small circular electrode $M O$. From the relation of the diffusional current and the rotating angles between the radius $M O$ and the rotating axis which is perpendicular to $M O$ the liquid velocity can be decided. This method is useful for the measurement of three-dimensional steady flow but is not suitable for unsteady flow or the measurement of

\footnotetext{
* Received on April 24, 1974

Presented at the 1st PACHEC, Kyoto 1972 (Preprints 18-a)

** Tokyo Univ. of Agriculture and Technology

于152 東京都目黒区大岡山2-12-1

東京工業大学工学部化学工学科 伊藤四郎
}

turbulence properties. Therefore, another new technique is required for this purpose.

In this paper, the principle of such a new method and an example of its application for measuring flow in a stirred vessel are described.

\section{Principle of Measurement}

When a certain voltage is applied between cathode and anode, the current is controlled by the rate of mass transfer of the reacting ions to the surface of the small polarized electrode. The diffusional current, $i_{d}$, is related to the velocity gradient, $\kappa$, at the surface of the electrode as follows ${ }^{2}$.

$$
i_{d}=A \kappa^{1 / 3}
$$

where

$$
A=0.721\left(C_{b}-C_{w}\right)\left(n F a D^{2 / 3}\right) / \Gamma(4 / 3) l^{1 / 3}
$$

The value of $A$ depends on the dimensions of the small electrode, that is, $a$ and $l$, the concentration, $C$, and the coefficient of molecular diffusion of the reacting species, $D$. Provided with diffusion-controlling conditions, the concentration of the reacting ions at the surface of the electrode, $C_{w}$, can be treated as essentially zero. The velocity gradient at the electrode located on the spherical surface can be

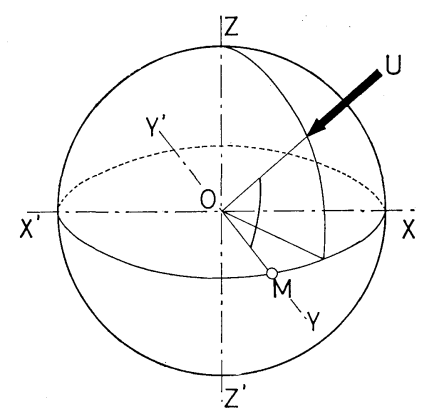

Fig. 1 Probe with one electrode 
calculated from boundary layer theory ${ }^{1)}$ as follows:

$$
\begin{aligned}
\kappa= & \left(\frac{\partial u}{\partial y}\right)_{y=0}=1.244 \sqrt{\frac{U^{3}}{R \nu}}\left(\alpha-0.3925 \alpha^{3}\right. \\
& \left.+0.0421 \alpha^{5}-0.0025 \alpha^{7}\right)
\end{aligned}
$$

where $\alpha$ denotes the central angle of the sphere between the flow direction and the radius through electrode, $U$ is the free stream velocity, $R$ is the radius of the sphere and $\nu$ is the kinematic viscosity of the fluid.

Consequently, the relation between diffusional current and the angle $\alpha$ and the velocity $U$ is obtained from Eq. (1) and Eq. (2) as follows.

$$
i_{d}=K U^{1 / 2} f(\alpha)
$$

where

$$
\begin{gathered}
K=0.776 \frac{C_{b} n F a D^{2 / 3}}{(R \nu)^{1 / 6} \Gamma(4 / 3) l^{1 / 3}} \\
f(\alpha)=\left(\alpha-0.3925 \alpha^{3}+0.0421 \alpha^{5}-0.0025 \alpha^{7}\right)^{1 / 3}
\end{gathered}
$$

Under a certain measurement condition, the factor $K$ takes a constant value and the relation between $i_{d}$ and $U^{1 / 2}$ must be linear for a constant angle $\alpha$.

In practice, it is difficult to calculate the factor $K$ in accordance with Eq. (4), but it is possible to determine it experimentally. However, the theoretical relationship shown in Eq. (4) is still useful to estimate the effects of electrode dimensions, concentration of solution and others on the value of $K$.

The function $f(\alpha)$ shows the angular characteristics of the spherical probe for the diffusional current. It takes a maximum value when $\alpha$ takes the value of 1.01 radian, and is zero at the stagnation point $(\alpha=0)$ and the separation point $(\alpha=1.91)$ as shown in Fig. 2. Actually, $f(\alpha)$ is a function of the Reynolds number and the orientation of the electrode with respect to the stream. However, this function becomes independent of the Reynolds number of sufficiently high velocities $\left.\left(30<\operatorname{Re}<10^{4}\right)^{4}\right)$, and is only a function of $\alpha$ which can be expressed in terms of angle $\theta$ in horizontal plane and the angle $\varphi$ in vertical plane for the flow direction.

Letting $\theta$ be the dihedral angle between flow plane and $Z O M$-plane, and $\varphi$ the angle between velocity and $X Y$-plane as shown in Fig. 3, the central angle of the sphere between velocity vector and any $n$-th electrode $\alpha_{n}$ is generally related to $\theta$ and $\varphi$ by the equations of spherical trigonometry as follows.

$\cos \alpha_{n}=-\sin \varphi \cdot \sin \varphi_{n}+\cos \varphi \cdot \cos \varphi_{n} \cdot \cos \left(\theta_{n}-\theta\right)$

where $\theta_{n}$ and $\varphi_{n}$ show the position of the $n$-th electrode.

From the two relations of Eq. (3) and Eq. (6), instantaneous velocity $U$ and flow direction $\theta, \varphi$ may be decided by measuring simultaneously the

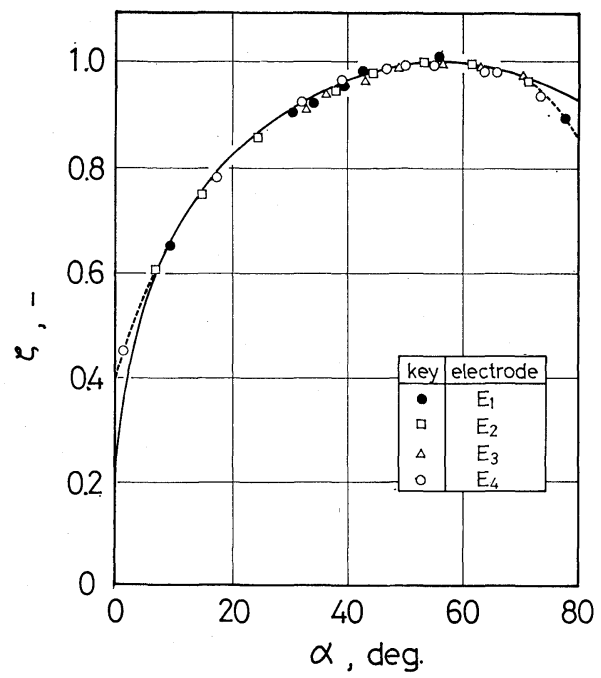

Fig. 2 Angular characteristic of probe

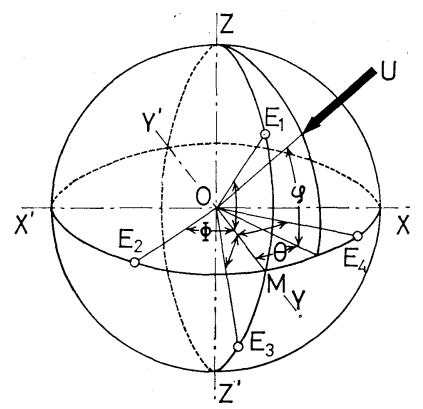

Fig. 3 Relation of flow direction and electrodes arrangement

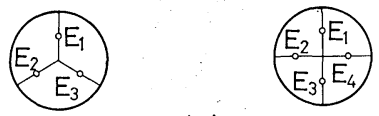

(b)

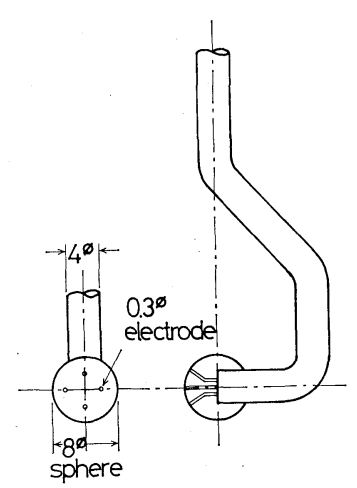

(a)

Fig. 4 Probes with multi-electrodes

diffusional current for each of the electrodes.

To decide $U, \theta$ and $\varphi$, it is required that the probe hold three or more electrodes. In this paper we shall consider the probe having three or four electrodes which are distributed symmetrically on the surface of the sphere and spaced at the same angular distance from the center point, $M$, as shown in Fig. 4 (b). 


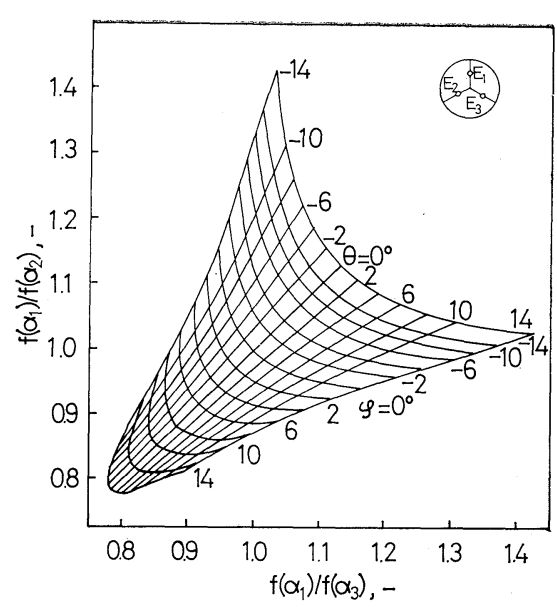

Fig. $5 \theta-\varphi$ diagram for three-electrode probe at $\Phi=\pi / 6$

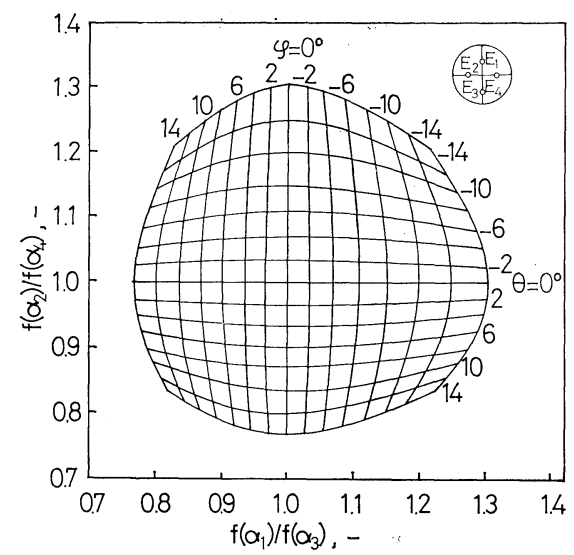

Fig. $6 \theta-\varphi$ diagram for four-electrode probe at $\Phi=\pi / 6$

\section{Probe with three electrodes}

Each of the three symmetrically spaced electrodes lies on a sphere surface and makes an angle, $\Phi$, with the center point, $M$, of the probe. If the central angles formed by each of the three electrodes and the flow direction are denoted as $\alpha_{1}, \alpha_{2}$ and $\alpha_{3}$ respectively, the following relations are obtained according to Eq. (6).

$\alpha_{1}=\cos ^{-1}\{\sin \varphi \cdot \sin \Phi+\cos \varphi \cdot \cos \Phi \cdot \cos \theta\}$

$\alpha_{2}=\cos ^{-1}\left\{-\sin \varphi \cdot \sin \varphi_{0}+\cos \varphi \cdot \cos \varphi_{0} \cdot \cos \left(-\theta_{0}-\theta\right)\right\}$

$\alpha_{3}=\cos ^{-1}\left\{-\sin \varphi \cdot \sin \varphi_{0}+\cos \varphi \cdot \cos \varphi_{0} \cdot \cos \left(\theta_{0}-\theta\right)\right\}$

where

$$
\begin{aligned}
& \theta_{0}=\tan ^{-1}\{\cos (\pi / 6) \cdot \tan \Phi\} \\
& \varphi_{0}=\sin ^{-1}\{\sin (\pi / 6) \cdot \sin \Phi\}
\end{aligned}
$$

The located positions of electrodes are given as $E_{1}(0, \Phi), E_{2}\left(-\theta_{0}, \varphi_{0}\right)$ and $E_{3}\left(\theta_{0}, \varphi_{0}\right)$ in a spherical coordinate with the axis $M O$.

On the other hand, from Eq. (3) the diffusional currents flowing through each of the three electrodes are given as

$$
\begin{aligned}
& i_{d 1}=K U^{1 / 2} f\left(\alpha_{1}\right) \\
& i_{d 2}=K U^{1 / 2} f\left(\alpha_{2}\right) \\
& i_{d 3}=K U^{1 / 2} f\left(\alpha_{3}\right)
\end{aligned}
$$

However, any electrode is required to have identical angular characteristics and sensitivity.

By combining Eq. (5) for each of the three electrodes, the following ratios may be obtained.

$$
\left.\begin{array}{rl}
\frac{i_{d 1}}{i_{d 2}} & =\frac{f\left(\alpha_{1}\right)}{f\left(\alpha_{2}\right)} \\
& =\left[\frac{\alpha_{1}\left\{1-\alpha_{1}^{2}\left(0.3925-0.0421 \alpha_{1}^{2}\right)\right\}}{\alpha_{2}\left\{1-\alpha_{2}^{2}\left(0.3925-0.0421 \alpha_{2}^{2}\right)\right\}}\right]^{1 / 3}=F_{12}(\theta, \varphi) \\
\frac{i_{d 2}}{i_{d 3}} & =\frac{f\left(\alpha_{2}\right)}{f\left(\alpha_{3}\right)} \\
& =\left[\frac{\alpha_{2}\left\{1-\alpha_{2}^{2}\left(0.3925-0.0421 \alpha_{2}^{2}\right)\right\}}{\alpha_{3}\left\{1-\alpha_{3}^{2}\left(0.3925-0.0421 \alpha_{3}^{2}\right)\right\}}\right]^{1 / 3}=F_{23}(\theta, \varphi)
\end{array}\right\}
$$

Each of these ratios may be determined experimentally by measuring the diffusional current of corresponding electrodes on the sphere. The terms of the ratio of $i_{d 1} / i_{d 2}$ and $i_{d 2} / i_{d 3}$ give implicitly $\theta$ and $\varphi$ which show the direction of velocity.

These ratios have finite values because $\alpha_{n}$ must be in the angular range of $0<\alpha_{n}<\pi / 3$, as shown in Fig. 2. The theoretical determination of the ratios of diffusional current may be based upon the angular characteristics of the probe.

The instantaneous direction of velocity can be determined by solving simultaneous equation (10) for $\theta$ and $\varphi$. When the flow direction $\theta, \varphi$ is decided, the magnitude of the instantaneous velocity may be determined by applying one of the diffusional current relations in Eq. (9), say, electrode $n$, as follows.

$$
U=\left[\frac{i_{d n}}{K f\left(\alpha_{n}\right)}\right]^{2}
$$

The diagram for determining the flow direction is obtained by the theoretical ratios of diffusional current as shown in Fig. 5, when $\Phi=\pi / 6$.

\section{Probe with four electrodes}

Similarly to the case of the three-electrode probe, let $\alpha_{1}, \alpha_{2}, \alpha_{3}$ and $\alpha_{4}$ be the central angles between each of the four electrodes and flow direction respectively, as shown in Fig. 3. When the located positions of the four electrodes are given as $E_{1}(0, \Phi), E_{2}(-\Phi, 0)$, $E_{3}(0,-\Phi)$ and $E_{4}(\Phi, 0)$ the equations equivalent to Eq. (7) are obtained as

$$
\begin{aligned}
& \alpha_{1}=\cos ^{-1}\{\sin \varphi \cdot \sin \Phi+\cos \varphi \cdot \cos \Phi \cdot \cos \theta\} \\
& \alpha_{2}=\cos ^{-1}\{\cos \varphi \cdot \cos (\Phi+\theta)\} \\
& \alpha_{3}=\cos ^{-1}\{-\sin \varphi \cdot \sin \Phi+\cos \varphi \cdot \cos \Phi \cdot \cos \theta\} \\
& \alpha_{4}=\cos ^{-1}\{\cos \varphi \cdot \cos (\Phi-\theta)\}
\end{aligned}
$$

The diffusional current at each of the four electrodes and their ratios are given by

$$
\left.\begin{array}{c}
i_{d 1}=K U^{1 / 2} f\left(\alpha_{1}\right) \\
i_{d 2}=K U^{1 / 2} f\left(\alpha_{2}\right) \\
i_{d 3}=K U^{1 / 2} f\left(\alpha_{3}\right) \\
i_{d 4}=K U^{1 / 2} f\left(\alpha_{4}\right)
\end{array}\right\}
$$


From the relationship of Eq. (13) and Eq. (14), the instantaneous values of $\theta, \varphi$ and $U$ are obtained continuously by measuring the diffusional current of the four electrodes in the same way as for the threeelectrode probe. These theoretical ratios of the diffusional current give the diagram for determining the flow direction shown in Fig. 6.

On comparing Fig. 6 with Fig. 5, it is evident that the diagram of the four-electrode probe is much less distorted and is more convenient for determining flow direction than that of the three-electrode probe.

When the values of $\theta, \varphi$ and $U$ are decided by the above-described way, the instantaneous three components of velocity are subsequently obtained.

Once a sufficient series of instantaneous velocity components have been made at any measuing point in the flow field, the three-dimensional mean velocities, the turbulent intensities and the double or triple correlations of velocity fluctuations can be caclulated statistically by making use of a computer.

This method has two inherent disadvantages, as the measuring angular range is limited $(-\pi / 9<\theta<\pi / 9$, $-\pi / 9<\varphi<\pi / 9)$ and the measurable turbulent scale is limited. Therefore, when the fluctuation of flow direction is very large, the probe must be adjusted along two perpendicular directions, for yaw and pitch, until the central point of the probe is opposed nearly to the mean flow direction. In addition, the measuring angular range can be extended by locating several more electrodes on the spherical surface. It is desirable that the probe have a diameter as small as possible to be capable of measuring small-scale turbulence and obtaining a rapid rate of response to the velocity fluctuations. But it is difficult to determine the response rate of the probe experimentally. According to Lighthill's analytical results ${ }^{3)}$ for the response of heat transfer to fluctuations in stream velocity, it may be roughly estimated that the probe will respond to frequencies up to $100 \mathrm{sec}^{-1}$ at the mean stream velocity $50 \mathrm{~cm} / \mathrm{sec}$.

\section{Calibration}

The probe used consisted of an 8 mm-diameter plastic sphere on which four circular platinum electrodes of $0.3 \mathrm{~mm}$ diameter are located symmetrically, as shown in Fig. 4(a). Each of the four electrodes makes an angle of 30 degrees with the center point of the probe. This probe was initially tested and calibrated in a shallow tank on a turn-table. The turn-table could be rotated in a horizontal plane by a variable-speed motor to obtain linear velocities up to $70 \mathrm{~cm} / \mathrm{sec}$. To measure the angular characteristics, the angle between each of the electrodes and the direction of turn-table motion was varied by yawing or pitching along two perpendi-

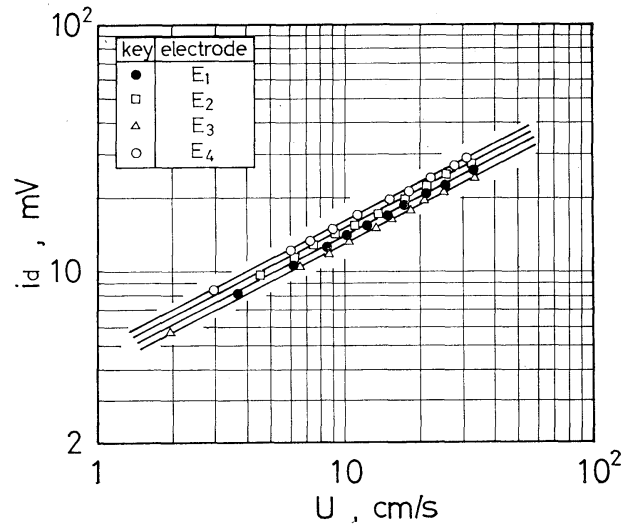

Fig. 7 Calibration curves

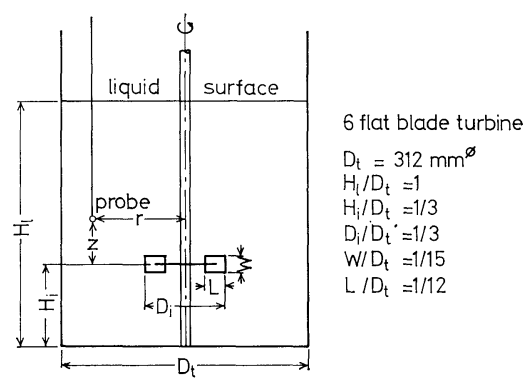

Fig. 8 Schema of stirred vessel used

cular directions.

The electrolytic reaction used was the oxidation of ferrocyanide ion on platinum electrodes.

$$
\mathrm{Fe}(\mathrm{CN})_{6}^{4-} \longrightarrow \mathrm{Fe}(\mathrm{CN})_{6}^{3-}+e
$$

The electrolyte was composed of $3 \times 10^{-3}$ mole $/ l$ potassium ferrocyanide and potassium ferricyanide and $1 \mathrm{~mole} / l$ potassium chloride.

An four electrodes and the large reference electrode, a $5 \times 5 \mathrm{~cm}$ platinum sheet, was +0.7 volt.

The experimental angular characteristics obtained in the way described above are shown in Fig. 2 by the dotted line. The experimental curve agrees well with the theoretical curve except near the stagnation point and to the rear of the maximum point. When the maximum diffusional current at $\alpha=1.01$ radian is plotted against the free-stream velocity on logarithmic graph paper, a linear relation is obtained as shown in Fig. 7.

Accordingly, the diffusional current, $i_{d}$, is proportional to $U^{1 / 2}$ as shown in the theoretical relation in Eq. (3).

\section{Application}

The flow field in an unbaffled stirred vessel was measured tentatively by this method.

The tank used was made of methacrylate plastic, of $312 \mathrm{~mm}$ inner diameter and $400 \mathrm{~mm}$ inner height, as shown in Fig. 8. The impeller was a standard turbine with six flat blades. 


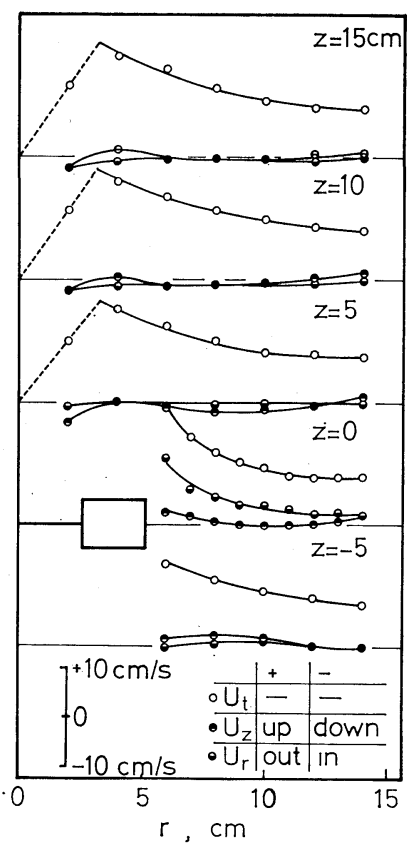

Fig. 9 Mean velocity distribution

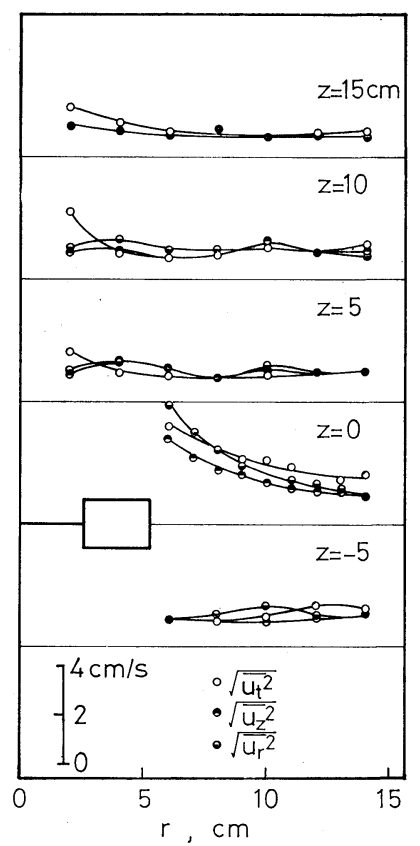

Fig. 10 Distribution of turbulent intensities

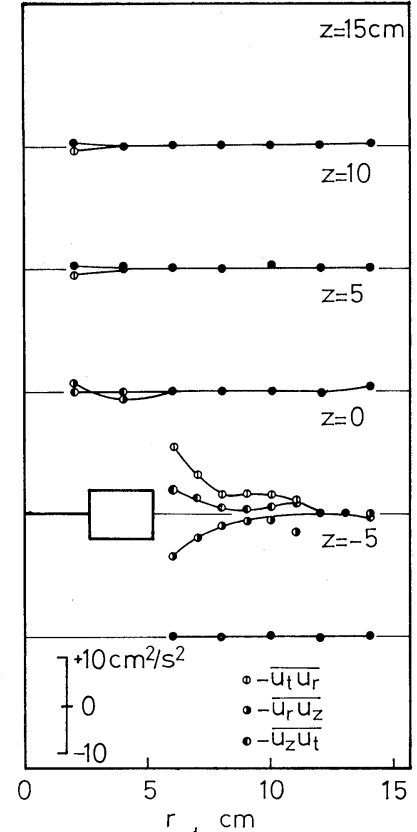

Fig. 11 Distribution of one-point double velocity correlations
At Reynolds number of $1.1 \times 10^{4}$, Part of the experimental results for the mean velocities, the turbulent intensities and the one-point double velocity correlations at five $z$-planes are shown in Figs. 9-11.

\section{Conclusions}

For measuring three-dimensional turbulence velocities, a new technique based on electrochemical reaction controlled by mass transfer rate is developed. The measuring probe for this method consists of a plastic sphere in which three or more polarized electrodes are plugged, their surfaces flush with the sphere surface. By making use of this measuring method, three-dimensional turbulence properties, that is, turbulent intensities, double and triple velocity correlations, etc., can be measured more correctly than by the traditional measuring method.

\section{Nomenclature}

$A$
$a$
$C$
$D$
$F$
$i$

$=$ constant in Eq. (1)

$=$ area of electrode surface

$=$ concentration of reacting species

$=$ coefficient of molecular diffusion

$=$ Faraday's constant

$=$ diffusional current $\left[\mathrm{cm}^{2}\right]$

[mole/l]

$\left[\mathrm{cm}^{2} / \mathrm{s}\right]$

[coulomb]

[A]
$K \quad=$ constant in Eq. (3)

$l \quad=$ effective length of electrode [cm]

$n \quad=$ number of electrons involved in electrode reaction

$\begin{array}{ll}R & =\text { radius of spherical probe } \\ U & =\text { free stream velocity }\end{array}$

$U_{t}, U_{r}, U_{z}=$ mean components of velocity

$u_{\iota}, u_{r}, u_{z} \quad$ fluctuating components of velocity $[\mathrm{cm} / \mathrm{s}]$ $[\mathrm{cm} / \mathrm{s}]$

$\alpha \quad=$ central angle between flow direction and electrode [radians, degrees]

$\Gamma \quad=\Gamma$ function $\quad[-]$

$\zeta \quad=i_{d} / i_{d \max }, \quad[-]$

$\theta \quad=$ dihedral angle between flow plane and $Z O M$-plane

$\kappa \quad=$ velocity gradient at wall $\quad[1 / \mathrm{s}]$

$\nu \quad=$ kinematic viscosity of fluid $\quad\left[\mathrm{cm}^{2} / \mathrm{s}\right]$

$\Phi=$ central angle between center point, $M$, and electrode

[radians]

$\varphi \quad=$ angle between velocity and $X Y$-plane [radians]

\section{Literature Cited}

1) Frössling, N.: Lunds. Univ. Arsskr, N. F. Avd., 2, 35, No. 4 (1940)

2) Ito, S. and S. Urushiyama: Kagaku Kögaku 32, 267 (1968)

3) Lighthill, M. J.: Proc. Roy. Soc. London, 224A, 1 (1954)

4) Thom, J.: A.R.C. Reports and Memoranda, No. 81176 (1928) 